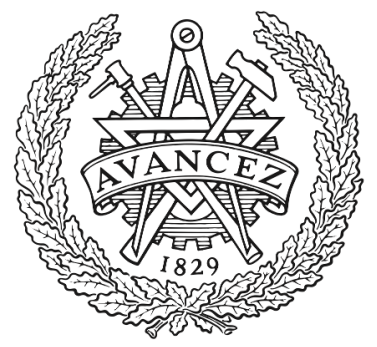

CHALMERS

UNIVERSITY OF TECHNOLOGY

\title{
Aggregation behavior of aqueous cellulose nanocrystals: the effect of inorganic salts
}

Downloaded from: https://research.chalmers.se, 2023-04-26 14:50 UTC

Citation for the original published paper (version of record):

Phan Xuan, T., Thuresson, A., Skepo, M. et al (2016). Aggregation behavior of aqueous cellulose nanocrystals: the effect of inorganic salts. Cellulose, 23(6): 3653-3663. http://dx.doi.org/10.1007/s10570-016-1080-1

N.B. When citing this work, cite the original published paper. 


\title{
Aggregation behavior of aqueous cellulose nanocrystals: the effect of inorganic salts
}

\author{
Tuan Phan-Xuan $(\mathbb{D} \cdot$ Axel Thuresson • Marie Skepö • Ana Labrador • Romain Bordes • \\ Aleksandar Matic
}

Received: 11 May 2016/Accepted: 26 September 2016/Published online: 30 September 2016

(C) The Author(s) 2016. This article is published with open access at Springerlink.com

\begin{abstract}
Natural anisotropic building-blocks such as cellulose nanocrystals (CNCs) have attracted considerable attention due to their biodegradability and nanometer-size. In this work the colloidal behavior of CNCs, obtained from sulfuric acid hydrolysis of microcrystalline cellulose, has been studied in presence of salts of different valences. The influence on the colloidal stability and nature of aggregates has been investigated for monovalent salts $(\mathrm{LiCl}, \mathrm{NaCl}, \mathrm{KCl}$, $\mathrm{CsCl})$, divalent salts $\left(\mathrm{CaCl}_{2}\right.$ and $\left.\mathrm{MgCl}_{2}\right)$, and a trivalent salt $\left(\mathrm{AlCl}_{3}\right)$, both experimentally by means of turbidity and small angle X-ray scattering (SAXS) measurements, as well as by Monte Carlo simulations using a simple coarse-grained model. For the entire salt series, a critical aggregation concentration (CAC) could be determined by turbidity measurements, as a result of the reduction of effective Coulomb repulsions
\end{abstract}

Electronic supplementary material The online version of this article (doi:10.1007/s10570-016-1080-1) contains supplementary material, which is available to authorized users.

T. Phan-Xuan $(\bowtie) \cdot$ A. Matic

Condensed Matter Physics, Department of Physics,

Chalmers University of Technology, Gothenburg, Sweden

e-mail: tuan.phan-xuan@chalmers.se

A. Thuresson · M. Skepö

Theoretical Chemistry, Department of Chemistry, Lund

University, Lund, Sweden

A. Labrador

MAX IV Laboratory, Lund, Sweden due to the presence of sulfate groups on the CNC surface. The CACs also followed the Schulze-Hardy law, i.e. the critical aggregation concentration decreased with increasing counterion valence. For the monovalent ions, the CACs followed the trend $\mathrm{Li}^{+}>\mathrm{Na}^{+}>\mathrm{K}^{+}>\mathrm{Cs}^{+}$, which could be rationalized in terms of matching affinities between the cation and the sulfate groups present at the surface of CNCs. From the SAXS measurements it was shown that the density of the aggregates increased with increasing salt concentration and ion valence. In addition, these findings were rationalized by means of simulation, which showed a good correlation with experimental data. The combination of the experimental techniques and the simulations offered insight into interactionaggregation relationship of CNC suspensions, which is of importance for their structural design applications.

\footnotetext{
R. Bordes

Applied Surface Chemistry, Department of Chemistry and Chemical Engineering, Chalmers University of Technology, Gothenburg, Sweden

T. Phan-Xuan $\cdot$ R. Bordes $(\bowtie)$

Vinn Excellence Center SuMo Biomaterials, Chalmers

University of Technology, Gothenburg, Sweden

e-mail: bordes@chalmers.se
} 
Keywords Cellulose nanocrystal - Aggregation · Hofmeister · Schulze-Hardy rule · Coarse-grained model $\cdot$ SAXS

\section{Introduction}

Anisotropic particles extracted from natural resources, such as cellulose nanocrystals (CNCs) or cellulose nanofibrils (CNFs), have many advantages of interest for material design. Recent reports have demonstrated that their use as innovative building blocks can lead to materials with outstanding performances (Håkansson et al. 2014). This mainly owes to the inherent characteristics of nanocellulose, such as high crystallinity and aspect ratio of the nanofibers (Peng et al. 2011). For instance, CNCs have been used for material reinforcement in nanocomposites and for designing functional nanomaterials towards special applications such as biomedical materials (drug carriers), optical materials, mechanically adaptive materials, emulsion stabilizers, decontamination of organic pollutants, etc. (Lin et al. 2012).

More recently, studies have focused on the interactions of CNCs in suspension and it is now regarded as a colloid rather than as a fiber. In that perspective, the aggregation and gelation of the nanocellulose dispersed in suspension become crucial for further development of new materials (Chau et al. 2015; Dufresne 2012; Salas et al. 2014). Thus, understanding how the interactions of nanocellulose, i.e. its colloidal behavior, depend on e.g. salt and/or polymer concentration is of great importance.

It is now well established that the stability of colloidal suspensions can be altered by the addition of salt, which affects the electrostatic contribution to the interparticle interactions. Already more than a century ago, Schulze and Hardy, found that salts containing multivalent ions were much more efficient in coagulating colloidal suspensions than monovalent ones (Lyklema 2013). This empirical observation was later rationalized by the DLVO theory, which assumes that the interaction potential of two colloidal particles is dominated by electrical double layer repulsions and van der Waals attractions. Suspensions of charged particles are therefore stable at low salt concentrations and the stability is lost when the salt concentration increases.
The transition between the stable dispersion and the aggregated state is rather sudden and it is referred to as the critical aggregation concentration (CAC). From the Schulze-Hardy rule, it can be shown that:

$C A C \propto \frac{1}{Z^{n}}$

where $\mathrm{Z}$ is the valence of the counterion, $\mathrm{n}=6$ for highly charged particles and $n=2$ for weakly ones. This rule has been proven to be valid for many systems of rod-like particles, such as carbon nanotubes (Sano et al. 2001) and nanocellulose (Rånby 1951; Wågberg et al. 2008).

Wågberg et al. (2008) and Fall et al. (2011), have developed a model for predicting colloidal stability of cellulose nanofibers (CNFs) bearing carboxyl groups on the surface based on DLVO theory. In their theory the coupled adsorption of protons and counterions with the carboxyl groups was also taken into consideration. This model describes how the stability of the $\mathrm{CNF}$ is affected as a function of $\mathrm{pH}$ and $\mathrm{NaCl}$ concentration. They have also demonstrated, experimentally, that aggregation and gelation are induced by decreasing the $\mathrm{pH}$, i.e. reducing the surface charge, or by increasing the salt concentration.

Similar alterations can be achieved by the addition of polymers (Borkovec and Papastavrou 2008), especially with polyelectrolytes (Borkovec et al. 2012). In Boluk et al. (2012) studied the aggregation behavior of $\mathrm{CNCs}$ in the dilute and the semi-dilute concentration range induced by the addition of water soluble polymers. They showed that the addition of rod shaped CNC particles in the semi-dilute regime led to the formation of weak gels and significantly altered the rheological and linear viscoelastic properties of the semi-dilute polymer solutions. They also proposed a model based on the DLVO theory with the introduction of a term dealing with the depletion forces between two parallel plates. Later, Fukuzumi et al. (2014) have studied TEMPO oxidized CNF in the dilute concentration regime in the presence of $\mathrm{NaCl}$, $\mathrm{MgCl}_{2}$ and $\mathrm{CaCl}_{2}$. They reported that the critical aggregation concentration of the rod particles could be explained by the interaction potential energy between two cylindrical rods based on DLVO theory.

Recently, the morphology of the CNC aggregates in the presence of $\mathrm{NaCl}$ was investigated in details using ultra small angle neutron scattering technique (Cherhal et al. 2015). The advantage of the technique is that 
it allows probing the aggregation process under a very large q-range. They have shown that at low salt concentration, the aggregates are formed by side-toside association of the CNC particles while for high salinity, they tend to organize in a randomly oriented configuration with a fractal dimension of about 2.1 for the charged CNCs and higher for the neutral system.

Despite a number of works on the stability of various type of nanocellulose (Araki 2013; Boluk et al. 2012; Cherhal et al. 2015; de Souza Lima et al. 2003; Dong et al. 1996; Elazzouzi-Hafraoui et al. 2008; Fukuzumi et al. 2014; Revol et al. 1992; Wågberg et al. 2008; Zhong et al. 2012), to the best of our knowledge, no systematic study has been reported on the stability of aqueous $\mathrm{CNC}$ suspensions as a function of electrolytes at different concentrations, and with ions of different valences. Furthermore, very little attention has been dedicated to the influence of these parameters on the structure of the formed aggregates. The structure of the aggregates and the concentration and valence dependence of the aggregation are central to understand when considering new materials from CNC suspensions.

In this work, CNCs stabilized by sulfate groups has been prepared by acid hydrolysis of microcrystalline cellulose. The aggregation of CNC particles in presence of salt of various valences was monitored by turbidity measurements, also taking into consideration the role of the Hofmeister serie for 1:1 salt, and by coarse-grained Monte Carlo simulations. The structural changes in the aggregation process were investigated by small angle $\mathrm{X}$-ray scattering.

\section{Materials, methods and simulation}

Materials

Dowex Marathon MR-3 hydrogen form (SigmaAldrich, Germany), $\mathrm{NaCl}$ (98.0\%, Sigma-Aldrich, Germany), $\mathrm{LiCl}$ (99.0 \%, Sigma-Aldrich, Germany), $\mathrm{CsCl}$ (99.0\%, Sigma-Aldrich, Germany), $\mathrm{KCl}$ (99.0\%, Sigma-Aldrich, Germany), $\mathrm{CaCl}_{2}$ (99.0\%, Sigma-Aldrich, Germany), $\mathrm{MgCl}_{2}$ (99.0\%, SigmaAldrich, Germany), $\mathrm{AlCl}_{3}$ (99.0\%, Sigma-Aldrich, Germany), and sulfuric acid (95.0-98.0\% w/w, Sigma-Aldrich, Germany), were used without further purification. Microcrystalline cellulose (Avicel PH$101 \mathrm{NF}$ ) was a donation from FMC BioPolymer, USA.
Water was purified with Millipore Milli-Q purification system (resistivity $>18.2 \mathrm{M} \Omega \mathrm{cm}$ ).

For the particle charge density titration a solution of $0.001 \mathrm{~N}$ polydiallyldimethyl ammonium chloride (polyDADMAC) was used.

Methods

\section{Preparation of cellulose nanocrystals}

The preparation route is adapted from the method reported by Bondeson et al. (2006). In brief, $40 \mathrm{~g}$ of MCC (Avicel PH-101 NF) was dispersed in Milli-Q water $(400 \mathrm{~mL})$ in a $2 \mathrm{~L}$ Erlenmeyer flask while stirred and cooled by an ice bath. Sulfuric acid (95-98\%, w/w) was added drop-wise to reach a final concentration of $64 \%(\mathrm{w} / \mathrm{w})$. The temperature was kept below $20{ }^{\circ} \mathrm{C}$ throughout the addition. The reaction mixture was then heated to $45{ }^{\circ} \mathrm{C}$, and left to react under vigorous stirring for $130 \mathrm{~min}$. Thereafter the reaction was quenched by an addition of $4 \mathrm{~L}$ deionized water and centrifuged at $3900 \mathrm{rpm}$ in cycles of $15 \mathrm{~min}$. The supernatant was discarded and replaced by deionized water. This procedure was repeated until the supernatant became turbid. The cellulose suspension was put on dialysis (membrane cut-off 12,000-14,000 Da) against deionized water, which was changed twice daily until the conductivity of deionized water was reached. The cellulose was then sonicated (Vibracell Sonicator, Sonics and Materials Inc., Danbury, CT, USA) at $40 \%$ output in three cycles of $14 \mathrm{~min}$ each. To ensure removal of any trace of ions, the cellulose suspension was ion-exchanged (Dowex Marathon MR-3 resin, hydrogen form) under continuous stirring for $48 \mathrm{~h}$. The mixture was filtered through a glass filter (no. 2) to separate the cellulose from the resin, and subsequently titrated by conductometry with a $\mathrm{NaOH}$ solution $(0.02 \mathrm{M})$. A final centrifugation step was carried out to remove any large aggregate (3900 rpm, $5 \mathrm{~min}$ ), resulting in suspension with a dry weight of $\sim 0.5 \%$ (w/w). The suspension was concentrated to $2 \%(\mathrm{w} / \mathrm{w})$ with a rotary evaporator.

Charge density titration was performed with a solution of polyDADMAC $0.001 \mathrm{~N}$ using a particle charge detector, CAS charge analyzing system (AFG, Analytic GMBH). The value of $0.20 \pm 0.02 \mathrm{e} / \mathrm{nm}^{2}$ is the average of 3 measurements. 


\section{Dynamic light scattering}

Depolarized and polarized dynamic light scattering (DDLS and DLS) measurements were performed on a ALV/CGS-8F (Germany). The light source was a solid-state laser, with a wavelength $\lambda=532 \mathrm{~nm}$, and the sample temperature was controlled by a thermostat bath to $20 \pm 0.2{ }^{\circ} \mathrm{C}$. Toluene has been used as an index matching liquid. Measurements were made at angles of observation $(\theta)$ between $12^{\circ}$ and $150^{\circ}$. The CNC particles were diluted in $10 \mathrm{mM} \mathrm{NaCl}$ to minimize the electrostatic interactions between rods.

The dynamic properties of the CNC suspension were investigated in the VV mode (vertical - vertical mode) as well as in the VH mode (vertical - horizontal mode) in order to probe the scattered intensity obtained from both rotational and translational motion. The intensity autocorrelation function (IACF) measured with DLS $\left(g_{2}(t)=I(0) I(t) / I^{2}\right)$ is related to the normalized electric field correlation function, $g_{1}(t)$, by the Siegert relation (Berne and Pecora 2000). $g_{1}(t)$ was analyzed in terms of a monomodal distribution of relaxation times:

$g_{1}(t)=\int A(\log \tau) \exp (-t / \tau) \mathrm{d} \log \tau$

where $\tau$ is the characteristic relaxation time.

\section{Small angle $X$ ray scattering}

Small angle X-ray scattering (SAXS) experiments were carried out at the I911-4 beamline (Labrador et al. 2013) at the MAX-IV laboratory synchrotron using a wavelength of $0.91 \AA$. The samples were analyzed in a capillary maintained at $20{ }^{\circ} \mathrm{C}$, and were prepared $24 \mathrm{~h}$ prior to the measurements. For each sample, the scattering of pure solvent (MilliQ water + salt) was also measured i.e. the background. Two-dimensional SAXS images were recorded in a PILATUS $1 \mathrm{M}$ detector (Dectris) using an exposure time of $120 \mathrm{~s}$. The scattering vector $q[q=4 \pi(\sin \theta) /$ $\lambda$, where $\lambda$ is the wavelength and $2 \theta$ is the scattering angle] was calibrated with a silver behenate sample. Reported scattering profiles $I(q)$ were obtained as the difference of the radial averaged SAXS 2D images from the sample and solvent. Data reduction was performed using the Nika package in Igor pro (Ilavsky 2012).

\section{Turbidity}

Turbidity measurements were performed as a function of wavelength in a rectangular airtight quartz cuvette with a path length of $10 \mathrm{~mm}$ using a Cary $60 \mathrm{UV}-\mathrm{Vis}$ spectrometer (Agilent Technologies). All the measurements reported in this study were performed at room temperature. The baseline was recorded by a measurement on MilliQ water, and the value of the absorbance at $400 \mathrm{~nm}$ was used as a measure of the turbidity in the samples. All samples were filtered before measurement using Sartorius $0.8 \mu \mathrm{m}$ filter before $2.5 \mathrm{~mL}$ of the sample was transferred to the cuvette. The titrations were carried out as follows: to $2.5 \mathrm{~mL}$ sample in a cuvette, 5-20 $\mu \mathrm{L}$ aliquots of a concentrated stock solution of salt were successively added, and after each addition the cuvette was shaken vigorously before measurements.

\section{Coarse-grained model}

We have used a coarse-grained model to study the interaction between two CNC rods, which is visualized in Fig. 1. The system consists of two charged rods, and its counterions, making it electroneutral. Due to computational resources, the size of the rods was reduced, although the aspect ratio was in agreement with the values determined by light scattering measurements. Each rod was modeled as nineteen overlapping and charged Lennard-Jones (LJ) spheres (sites), giving a total length, $L=34 \mathrm{~nm}$, and a diameter of $d=3.4 \mathrm{~nm}$. This is approximately five times smaller than experimental estimations, but there were no significant effects of the reduced size of the rods (c.f. simulations for a system with $L=17 \mathrm{~nm}$ and $\mathrm{d}=1.7 \mathrm{~nm})$.

The effect of water was taken into account implicitly, i.e. treated as a uniform continuum with a dielectric constant of $\epsilon_{r}=78.3$. All the interactions were assumed to be pairwise additive. The Hamiltonian of the system consists of two terms, (1) LennardJones (LJ) and (2) coulomb interaction, see Eq. (3)

$u_{i j}\left(r_{i j}\right)=4 \varepsilon_{i j}\left(\left(\frac{\sigma_{i j}}{r_{i j}}\right)^{12}-\left(\frac{\sigma_{i j}}{r_{i j}}\right)^{6}\right)+\frac{e^{2} z_{i} z_{j}}{4 \pi \epsilon_{0} \epsilon_{r} r_{i j}}$,

where $\varepsilon_{i j}$ determines the short-ranged van der Waals (vdW) attraction, $r_{i j}$ is the distance, and $\sigma_{i j}=\left(\sigma_{i}+\right.$ $\left.\sigma_{j}\right) / 2$ is the diameter for particle $i$ and $j$. The strength 


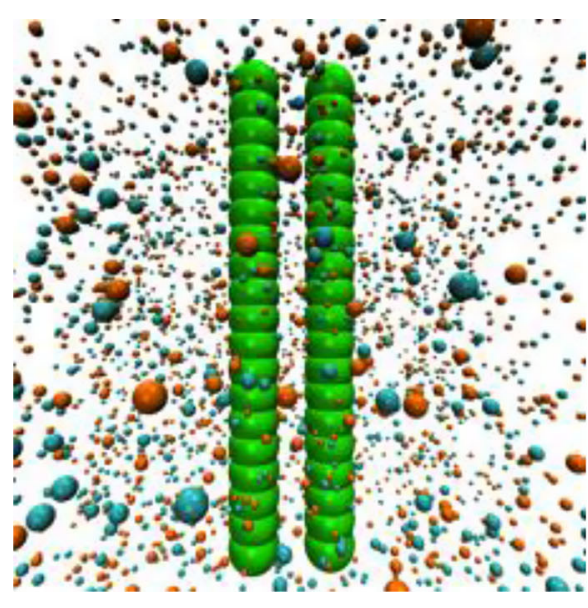

Fig. 1 Two illustrative snapshots with the salt concentrations $50 \mathrm{mM} \mathrm{NaCl}$ (left) and $0.2 \mathrm{mM} \mathrm{AlCl}_{3}$ (right). The rods (shown in green) are parallel, and the centers of mass are fixed on the $z$ axis. The smaller spheres corresponds to the ions that are free to

of the interaction parameter was set to $\varepsilon_{\text {ion-site }}=$ $\varepsilon_{i o n-i o n}=0.01 \mathrm{kT}$ and $\sigma_{\text {site }}=3.4 \mathrm{~nm}, \sigma_{\text {ion }}=0.4 \mathrm{~nm}$. $k, T, e, \epsilon_{0}$, and $z_{i}$ is the Boltzmann constant, temperature, elementary charge, permittivity of vacuum and valence of particle $i$, respectively.

\section{Monte Carlo simulations}

All particles were enclosed in a cylinder with length $L_{c y l}$ and radius $R_{c y l}$, and the volume of the cylinder was set to mimic the experimental concentrations. The two parallel CNC rods were fixed symmetrically around the origin with their center of mass on the z-axis, and the ions were allowed to explore the cell with random single particle translations. The equilibrium properties of the system were obtained by utilizing the Monte Carlo Metropolis simulation (Metropolis et al. 1953) within the grand canonical $(\mu \mathrm{VT})$ ensemble i.e. the chemical potential, volume, and temperature were kept constant. Thus, the salt was allowed to exchange with a bulk reservoir with a set chemical potential. All simulations were performed using Faunus (Stenqvist et al. 2013) and the temperature was set to $300 \mathrm{~K}$.

The free energy between the two parallel rods, $W(r)$, was calculated as a function of the center of mass distance, $r$, by integrating the mean force $\bar{F}_{z}\left(r^{\prime}\right)=F_{z}\left(r^{\prime} ; \boldsymbol{r}^{n}\right)$ in the z-direction between the rods: $W(r)=-\int_{-\infty}^{r} \bar{F}_{z}\left(r^{\prime}\right) \mathrm{d} r$, where $\boldsymbol{r}^{n}$ represents the coordinates of the freely moving $n$ ions. The mean force

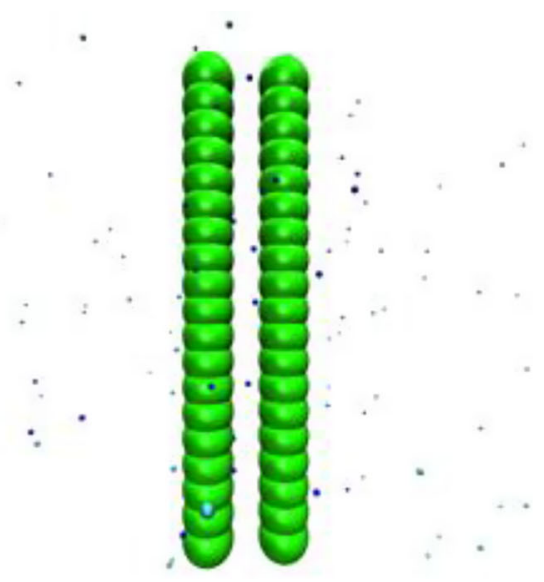

explore the cell, and they are shown in the following colour code: $\mathrm{Na}^{+}$- (orange), $\mathrm{Cl}^{-}$- (cyan), and $\mathrm{Al}^{3+}$-ions (blue). Note that objects which are nearby are illustrated larger than those far away

was evaluated at 40 different values of $r$ ranging from $3 \mathrm{~nm}$ to $14 \mathrm{~nm}$ using the mid-plane approach (Guldbrand et al. 1984) and the trapezoidal rule was used for integration.

The interaction between the rods was investigated for different surface charge densities, salt concentrations, and cation valences. In the model described above there are two fitting parameters: (1) the rod surface charge density, and (2) the non-electrostatic LJ-parameter between the rod sites. The probability of aggregation, $P_{a g g}$, was estimated by the following equation:

$P_{a g g}=e^{-U_{a g g}} /\left(e^{-U_{a g g}}+e^{-U_{n o n-a g g}}\right)$

where $U_{\text {agg }}$ is the free energy in $k T$ for the aggregated state i.e. at $\mathrm{r}=3.7 \mathrm{~nm}$ and $U_{\text {non-agg }}=0 \mathrm{kT}$ is the free energy in the non-aggregated state. Thus, the partition function is reduced to only include two configurations i.e. the non-aggregated and the aggregated states.

\section{Results and discussion}

Preparation and characterization of $\mathrm{CNCs}$

Cellulose nanocrystals were produced by hydrolysis of microcrystalline cellulose by sulfuric acid yielding negatively charged CNC particles with sulfate surface groups. This provided a stable dispersion of the particles in water as a consequence of the electrostatic 
repulsion. Polarized and depolarized dynamic light scattering have been used to characterize CNCs in salt free condition. Data analysis was based on the method described by de Souza Lima and Borsali (2004) and the detailed analysis can be found in Supporting Information, as well as results of static light scattering. The rotational and translational diffusion coefficients of the CNCs were determined form the experiment and from this data an average length of $\mathrm{L}=170 \mathrm{~nm}$ and a cross-section diameter of $\mathrm{d}=17 \mathrm{~nm}$ were calculated. These results are in good agreement with the literature values (Moon et al. 2011), and with the results obtained by AFM (see supporting information, Fig. S4).

\section{Aggregation behavior}

The aggregation behavior of the CNC suspension was studied by means of turbidity and SAXS. Simulations were conducted to reveal the role of salt concentration on the aggregation and qualitatively estimate the aggregation parameters.

\section{Influence of the CNC concentration}

The evolution of the absorbance at a wavelength $\lambda=400 \mathrm{~nm}$ was used to measure the turbidity of the solutions at different $\mathrm{CNC}$ concentrations, at different salt concentrations, and valence of the salts. As can be seen in Fig. 2a, for $\mathrm{NaCl}$ and $\mathrm{CaCl}_{2}$, the behavior of the turbidity versus salt concentration follows a classical colloidal behavior (Gregory 1976). After an initial plateau at low concentrations, where the presence of salt does not affect the colloidal behavior, a steep increase can be noticed. The concentration where the onset of the turbidity is observed is referred to as the critical aggregation concentration (CAC) and corresponds to the first macroscopic sign of aggregation. For concentrations greater than $\mathrm{CAC}$, the turbidity is increasing until a second plateau is reached. As visible, the CAC does not depend on the particle concentration, which confirms that the concentration range used throughout this study allows a direct observation of the balance of the attractive and repulsive forces in the system. At higher concentrations, aggregation would have induced a change in viscosity of the suspension leading to a strong concentration-dependence of the aggregation. This is obviously not the case here.

Figure $2 b$ shows the data normalized in absorbance and plotted against ionic strength instead of salt concentration. One can note that there is a clear difference in the onset of turbidity for the monovalent salt compared to the divalent salt. This indicates that it is not a simple screening effect of the electrostatic repulsive forces that is triggering the aggregation. In such a situation, the value of CAC would be nearly identical. This will be discussed further below.

\section{Ion specific effects for monovalent ions}

As can be seen in Fig. 3a, for monovalent salts the CAC follows the order: $\mathrm{Li}^{+}>\mathrm{Na}^{+}>\mathrm{K}^{+}>\mathrm{Cs}^{+}$. The trend for the CAC for monovalent salts cannot be explained solely on the basis of simple coulombic interactions and more complex effects such as ion specificity, where ions of different size but of the same valence do not interact in the same manner with a charged surface, have to be taken into account (Lo Nostro and Ninham 2012; Ninham and Lo Nostro
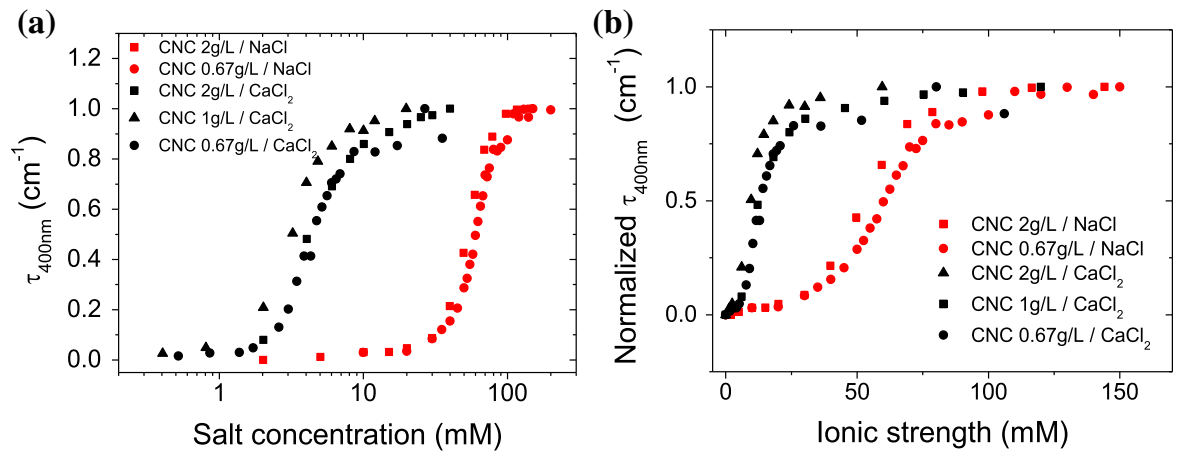

Fig. 2 Turbidity at $\lambda=400 \mathrm{~nm}$ of CNC suspension for different CNC concentrations as a function of (a, left) salt concentration and of (b, right) ionic strength for divalent salts and monovalent salts, as indicated in the figure 


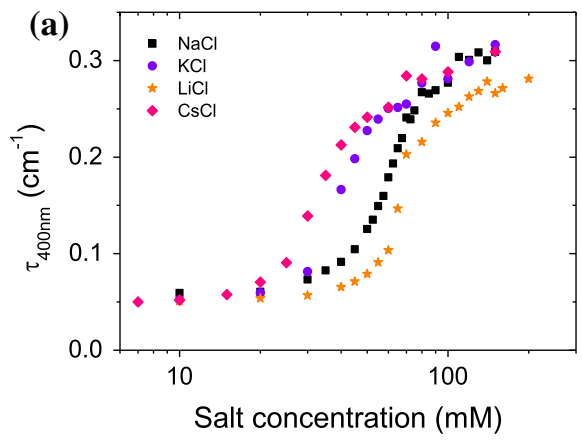

Fig. 3 (a, left) Turbidity at $\lambda=400 \mathrm{~nm}$ of CNC suspension at $\mathrm{C}=0.67 \mathrm{~g} / \mathrm{L}$ as a function of salt concentration for monovalent salts. (b, right) Turbidity at $\lambda=400 \mathrm{~nm}$ of CNC suspension at

2010; Vlachy et al. 2009). Similar effects have been observed for other colloids such as bubbles (Craig et al. 1993), silica nanoparticles (Franks 2002) as well as globular proteins (Boström et al. 2003). Our results show a direct correspondence to the Hofmeister series where the surface affinity increases according to $\mathrm{Li}^{+}>\mathrm{Na}^{+}>\mathrm{K}^{+}>\mathrm{Cs}^{+}$. The correspondence between the $\mathrm{CAC}$ and the surface affinity can be rationalized since the ions that adsorb close to the surface will have a high screening efficiency; consequently, a lower electrolyte concentration is required to destabilize the dispersion (Lyklema 2003, 2009). A tentative explanation for the surface affinity can be obtained from the law of matching affinities, proposed by Vlachy et al. (2009). As can be seen in Fig. 3a, the lowest CAC was determined for soft cations such as Cesium whereas it increased significantly with Lithium (hard cations). Owing to the preparation route, the stability of CNC particles is due to the presence of charged sulfate groups, which are also soft in terms of charge density. There is a good affinity between cesium and sulfate groups, while lithium interacts to less extent with the CNC surface charges. As a result, the aggregation is triggered at lower concentration for Cesium than for Lithium.

\section{Influence of the ion valence}

In systems where solely electrostatic interactions are involved in the stabilization, a strong dependency in the CAC can be observed with the valence of the ions. We therefore carried out a systematic series of turbidity measurement at a fixed $\mathrm{CNC}$ concentration $(\mathrm{C}=0.67 \mathrm{~g} / \mathrm{L})$ for different salts, lithium, sodium,

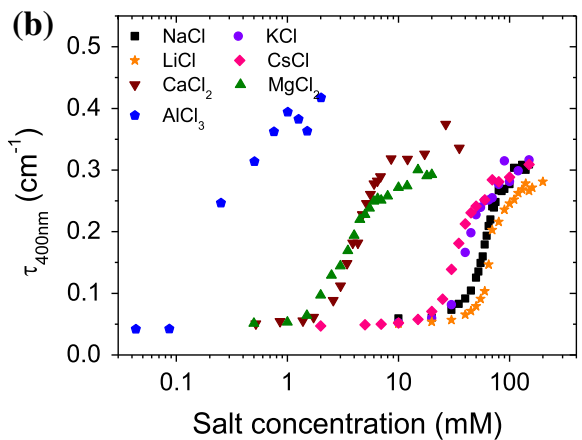

$\mathrm{C}=0.67 \mathrm{~g} / \mathrm{L}$ as a function of salt concentration for monovalent, divalent, and trivalent salts

potassium and cesium chloride for the monovalent species, calcium and magnesium chloride for the divalent species, and aluminum chloride for the trivalent species, to reveal the effect of the valence of the ions. The results are shown Fig. 3b. As can be seen, the CAC shifts by one order of magnitude when increasing the valence of the cation by one. Such a strong effect on the CAC of colloidal systems is usually approximated by the Schulze-Hardy rule in terms of $1 / Z^{6}$, where $\mathrm{z}$ is the valence of the cation (Lyklema 2013). Our data follows well the SchulzeHardy rule, even though a minor deviation can be observed, see supporting information. A slight deviation from the Schulze-Hardy behavior can easily be expected since the current system deals with nonspherical particles. Furthermore this rule is often regarded as a rule of thumb since it is based on several approximations.

Internal structure of the aggregate

The internal structure of CNC aggregates at $\mathrm{C}=0.67 \mathrm{~g} / \mathrm{L}$ formed by adding salt was investigated by means of SAXS. Figure 4 shows an example of the experimental data at three selected concentrations of the monovalent salt $\mathrm{KCl}$. No correlation peak could be detected for the investigated $q$-range, and solely a variation in the slope of the scattering curve for $q \leq 0.05 \AA^{-1}$ was observed. This indicates that the aggregates are randomly oriented with an internal structure that is independent of salt concentration.

In the investigated $q$-range, the scattering intensity follows a power-law dependence as function of $q$, which indicates that the internal structure is fractal 


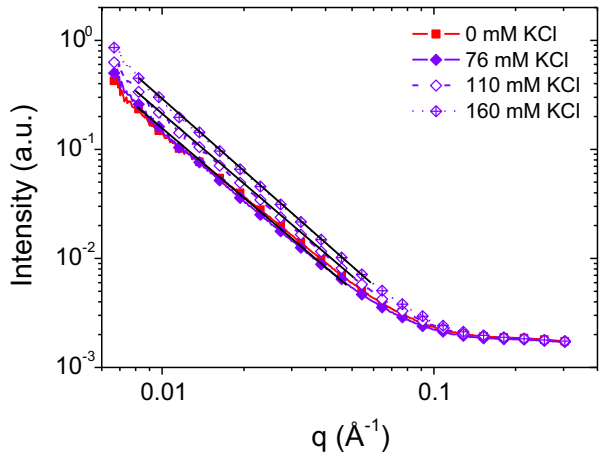

Fig. 4 Logarithmic SAXS curves of CNC aggregates for different $\mathrm{KCl}$ concentrations at a fixed $\mathrm{CNC}$ concentration $(\mathrm{C}=0.67 \mathrm{~g} / \mathrm{L})$. Solid lines represent a power law fit where the slope is the fractal dimension of the nanostructure of the aggregates

(Sorensen 2001). The fractal dimension $d_{f}$ can be determined from the slope of the curve, and it is directly related to the compactness of the aggregates, where densely packed structures result in an increase in the scattering intensity and a steeper decay compared to more open structures with smaller fractal dimensions. Figure 5 shows the fractal dimension as a function of the salt concentration. The fractal dimension increases with increasing salt concentration, corresponding to a densification of the aggregates. One can also observe that there is a rather strong dependence on the valence of the cations with a decrease in the onset of densification with increased valence. Similar behavior was found in CNC hydrogel systems for higher rod concentration (Chau et al. 2015), where it was shown that the mesh size of the gels increased with increasing the salt concentration or by increasing $\mathrm{CNC}$ concentration at constant salt

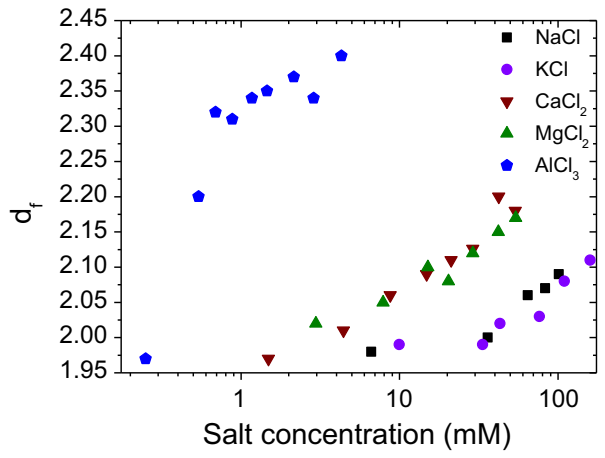

Fig. 5 Fractal dimension as a function of salt concentrations for different salts at a CNC concentration of $0.67 \mathrm{~g} / \mathrm{L}$ content. Furthermore, the maximum values of $d_{f}$ increase with increasing the ion valence, from $d_{f}=2.1$ for monovalent ions to $d_{f}=2.15$ for divalent and $d_{f}=2.35$ for trivalent ions. The values for monovalent ions was in good agreement with the ones reported by Cherhal et al. (2015) where similar values of $d_{f}$ were found in the case of charged CNCs. Interestingly, the value of $d_{f}=2.35$ obtained from trivalent ion is slightly higher than the one from the neutral CNCs system in the presence of monovalent ions where the aggregates have a value of 2.3.

The results for the fractal dimension correlate with the results for the CAC, both showing a strong increase at a certain salt concentration and reaching a maximum value at high salt concentrations. The agreement between the onset in turbidity (CAC) and slope increase (fractal dimension) in the SAXS experiment with increasing salt concentration, as well as the slope increase with cation valence as a function salt concentration, have previously been found for the clay mineral montmorillonite (Michot et al. 2013). Although the slope is larger for clay, it agrees well with the general behavior for colloids driven mainly by electrostatic interactions.

\section{Monte Carlo simulations}

By utilizing a coarse-grained model, we could obtain a molecular understanding of the aggregation properties of the system, and how it was affected by ionic strength and valence of the cations. The experimental CACs were estimated to be $50 \mathrm{mM} \mathrm{NaCl}, 5 \mathrm{mM}$ $\mathrm{CaCl}_{2}$, and $0.2 \mathrm{mM} \mathrm{AlCl}_{3}$ (see Fig. 3). These results have been used to estimate the first fitting parameter (the surface charge density) of the CNC rods, where the CAC should correspond to a global minimum in the free energy. In order to observe such behavior for our model system, we investigated the effect of pure electrostatic interactions with the aim to find a rod surface charge density that gave the same free energies at molecular contact i.e. the aggregated state for all the above given salts.

The free energies for several different surface charge densities were calculated, and for a surface charge density of $-0.1 \mathrm{e} / \mathrm{nm}^{2}$, similar free energies between the different salts were found, i.e. at molecular contact $3.7 \mathrm{~nm}$. Such a surface density is in agreement with values found in the literature (Kalashnikova et al. 2012), even though larger values can also 
(a)

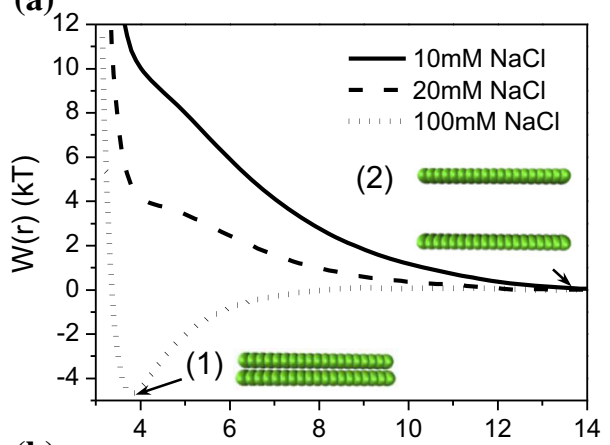

(b)
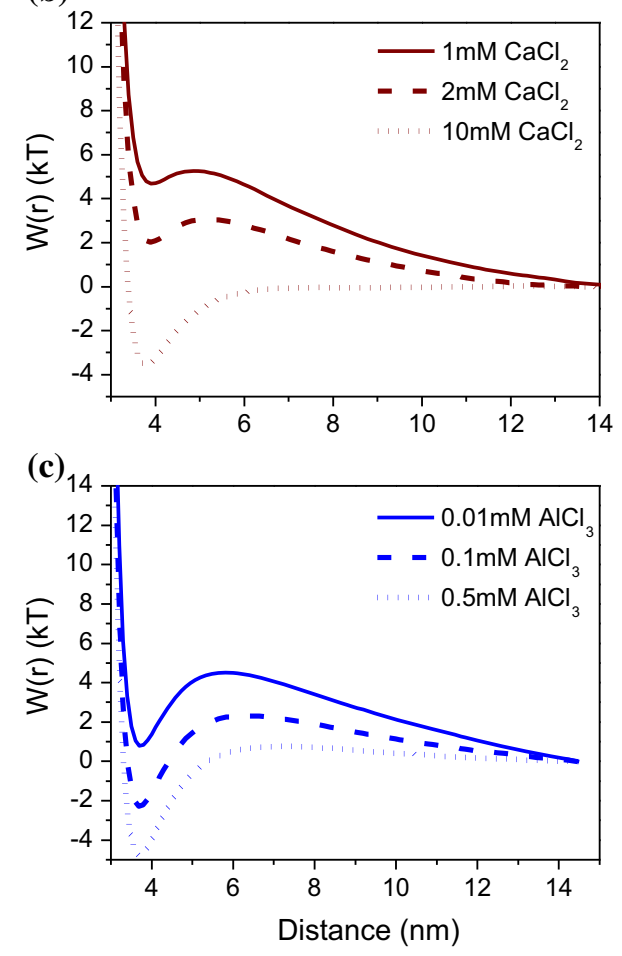

Fig. 6 Free energy as a function of center of mass distance, $r$, between two parallel rods with surface charge density $-0.1 \mathrm{e} /$ $\mathrm{nm}^{2}$ and $\varepsilon_{\text {site-site }}=0.13 \mathrm{kT}$. a Free energy for three different concentrations of $\mathrm{NaCl}(10,20$, and $100 \mathrm{mM})$. Two separations between the rods are highlighted, (1) corresponds to the aggregated state $(r=3.7 \mathrm{~nm})$ and (2) corresponds to a stabilized, non-aggregated state. b Free energy for three different concentrations of $\mathrm{CaCl}_{2}(1,2$, and $10 \mathrm{mM})$. c Free energy for three different concentrations of $\mathrm{AlCl}_{3}(0.01,0.1$, and $0.5 \mathrm{mM}$ )

be found, as experimentally determined here. The long-ranged electrostatic repulsion increased in the following order $50 \mathrm{mM} \mathrm{NaCl}<5 \mathrm{mM} \mathrm{CaCl}_{2}<0.2$ $\mathrm{mM} \mathrm{AlCl}$, and for the short-ranged electrostatic repulsion, we found the opposite behavior. The screening of the electrostatic interaction alone did not induce aggregation; therefore we had to tune the second fitting parameter $\varepsilon_{\text {site-site }}$ (the vdW attractive interaction) to observe aggregation. To obtain a global free energy minimum between the rods for the selected salt concentrations, an $\varepsilon_{\text {site-site }}=0.13 \mathrm{kT}$ was introduced. A higher charge density can be compensated by stronger $\mathrm{vdW}$ interaction, i.e. a higher $\varepsilon_{\text {site-site }}$ value.

In Fig. 6 the free energy between the rods (rod surface charge density of $-0.1 \mathrm{e} / \mathrm{nm}^{2}$ ) is shown for several salt concentrations.

Figure 7 shows the probability of aggregation as a function of salt concentration and ionic strength. We found a high probability of aggregation at $100 \mathrm{mM}$ $\mathrm{NaCl}, 10 \mathrm{mM} \mathrm{CaCl}_{2}$, and $0.1 \mathrm{mM} \mathrm{AlCl}$. The probability of aggregation in Fig. 7 was very well correlated to the onset of turbidity (i.e. the CAC) shown in Fig. 2 and Fig. $3 b$.

\section{Conclusions}

This work presents in detail the colloidal stability and aggregation behavior of aqueous CNC suspensions in the presence of salts with different valences. These anisotropic particles were perfectly stabilized at low ionic strength, which gave us a possibility to determine particle dimension using light scattering techniques, while they tended to aggregate with increasing salt content, resulting in an increase of the turbidity. Multivalent ions were much more efficient in inducing aggregation than the monovalent ones, which resulted in a decrease of CAC with increasing cation valence. However, the CAC did not perfectly follow the Schulze-Hardy rule, which indicated that, other interactions than the electrostatic screening effect probably were involved in controlling the observed behavior. The density of the aggregates increased with increasing salt concentration above the CAC and became constant at high salt concentrations.

A coarse-grained model for cellulose nanocrystals that includes the vdW-forces and the electrostatic interactions was used to analyze the effect of valence and salt concentration. The model had two fitting parameters: the surface charge density and the strength of the vdW-forces. For a surface charge density of $-0.1 \mathrm{e} / \mathrm{nm}^{2}$ and an $\varepsilon_{\text {site-site }}=0.13 \mathrm{kT}$, the simulations agreed well with the experiments where the probability of aggregation was comparable to the turbidity measurements. 

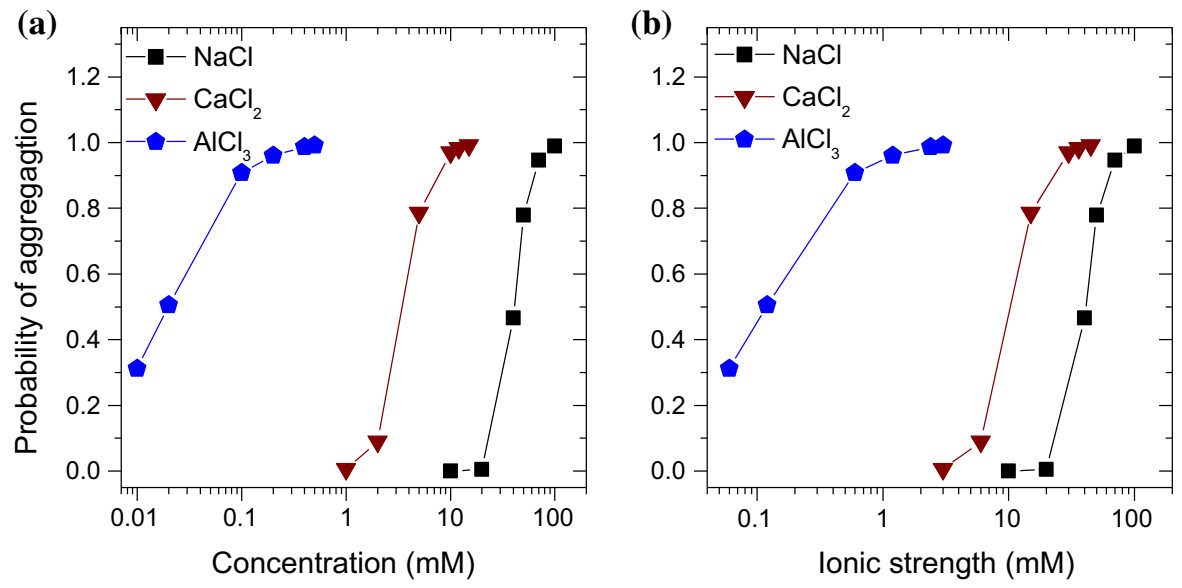

Fig. 7 Probability of aggregation as a function of $\mathbf{a}$ salt concentration and $\mathbf{b}$ ionic strength according to Eq. (4)

The results from this study are of importance for controlling the aggregation and gelation of the CNCs thus offering new opportunities for structural design of this new class of anisotropic particles.

Acknowledgments MAX IV Laboratory is acknowledged for providing access to the facility. Computational resources were provided by the Swedish National Infrastructure for Computing (SNIC) through Lunarc, the Center for Scientific and Technical Computing at Lund University. The computational work was performed within the framework of the Swedish national strategic e-science research program eSSENCE. Dr. Martin Turesson is acknowledged for fruitful and valuable discussions.

Open Access This article is distributed under the terms of the Creative Commons Attribution 4.0 International License (http:// creativecommons.org/licenses/by/4.0/), which permits unrestricted use, distribution, and reproduction in any medium, provided you give appropriate credit to the original author(s) and the source, provide a link to the Creative Commons license, and indicate if changes were made.

\section{References}

Araki J (2013) Electrostatic or steric? Preparations and characterizations of well-dispersed systems containing rod-like nanowhiskers of crystalline polysaccharides. Soft Matter 9:4125-4141. doi:10.1039/C3sm27514k

Berne BJ, Pecora R (2000) Dynamic light scattering: with applications to chemistry, biology, and physics. Dover Books on Physics, Dover edn. Dover Publications, Mineola

Boluk Y, Zhao LY, Incani V (2012) Dispersions of nanocrystalline cellulose in aqueous polymer solutions: structure formation of colloidal rods. Langmuir 28:6114-6123. doi:10.1021/La2035449

Bondeson D, Mathew A, Oksman K (2006) Optimization of the isolation of nanocrystals from microcrystalline cellulose by acid hydrolysis. Cellulose 13:171-180. doi:10.1007/ s10570-006-9061-4

Borkovec M, Papastavrou G (2008) Interactions between solid surfaces with adsorbed polyelectrolytes of opposite charge. Curr Opin Colloid Interface Sci 13:429-437. doi:10.1016/ j.cocis.2008.02.006

Borkovec M, Szilagyi I, Popa I, Finessi M, Sinha P, Maroni P, Papastavrou G (2012) Investigating forces between charged particles in the presence of oppositely charged polyelectrolytes with the multi-particle colloidal probe technique. Adv Colloid Interface Sci 179-182:85-98. doi:10.1016/j.cis.2012.06.005

Boström M, Williams DRM, Ninham BW (2003) Specific ion effects: why the properties of lysozyme in salt solutions follow a hofmeister series. Biophys J 85:686-694. doi:10. 1016/S0006-3495(03)74512-3

Chau $\mathrm{M}$ et al (2015) Ion-mediated gelation of aqueous suspensions of cellulose nanocrystals. Biomacromolecules 16:2455-2462. doi:10.1021/acs.biomac.5b00701

Cherhal F, Cousin F, Capron I (2015) Influence of charge density and ionic strength on the aggregation process of cellulose nanocrystals in aqueous suspension, as revealed by small-angle neutron scattering. Langmuir 31:5596-5602. doi:10.1021/acs.langmuir.5b00851

Craig VSJ, Ninham BW, Pashley RM (1993) Effect of electrolytes on bubble coalescence. Nature 364:317-319. doi:10.1038/364317a0

de Souza Lima MM, Borsali R (2004) Rodlike cellulose microcrystals: structure, properties, and applications. Macromol Rapid Commun 25:771-787. doi:10.1002/marc. 200300268

de Souza Lima MM, Wong JT, Paillet M, Borsali R, Pecora R (2003) Translational and rotational dynamics of rodlike cellulose whiskers. Langmuir 19:24-29. doi:10.1021/ $\mathrm{La} 020475 \mathrm{z}$

Dong XM, Kimura T, Revol JF, Gray DG (1996) Effects of ionic strength on the isotropic-chiral nematic phase transition of suspensions of cellulose crystallites. Langmuir 12:2076-2082. doi:10.1021/la950133b

Dufresne A (ed) (2012) Rheological behavior of nanocellulose suspensions and self-assembly. In: Nanocellulose: from 
nature to high performance tailored materials. Walter de Gruyter, Berlin

Elazzouzi-Hafraoui S, Nishiyama Y, Putaux JL, Heux L, Dubreuil F, Rochas C (2008) The shape and size distribution of crystalline nanoparticles prepared by acid hydrolysis of native cellulose. Biomacromolecules 9:57-65. doi:10.1021/Bm700769p

Fall AB, Lindström SB, Sundman O, Ödberg L, Wågberg L (2011) Colloidal stability of aqueous nanofibrillated cellulose dispersions. Langmuir 27:11332-11338. doi:10. 1021/la201947x

Franks GV (2002) Zeta potentials and yield stresses of silica suspensions in concentrated monovalent electrolytes: isoelectric point shift and additional attraction. J Colloid Interface Sci 249:44-51. doi:10.1006/Jcis.2002.8250

Fukuzumi H, Tanaka R, Saito T, Isogai A (2014) Dispersion stability and aggregation behavior of TEMPO-oxidized cellulose nanofibrils in water as a function of salt addition. Cellulose 21:1553-1559. doi:10.1007/S10570-0140180-Z

Gregory J (1976) The effect of cationic polymers on the colloidal stability of latex particles. J Colloid Interface Sci 55:35-44. doi:10.1016/0021-9797(76)90006-0

Guldbrand L, Jonsson B, Wennerstrom H, Linse P (1984) Electrical double-layer forces-a Monte-Carlo study. J Chem Phys 80:2221-2228. doi:10.1063/1.446912

Håkansson KMO et al (2014) Hydrodynamic alignment and assembly of nanofibrils resulting in strong cellulose filaments. Nat Commun. doi:10.1038/ncomms5018

Ilavsky J (2012) Nika: software for two-dimensional data reduction. J Appl Crystallogr 45:324-328. doi:10.1107/ S0021889812004037

Kalashnikova I, Bizot H, Cathala B, Capron I (2012) Modulation of cellulose nanocrystals amphiphilic properties to stabilize oil/water interface. Biomacromolecules 13:267-275. doi:10.1021/bm201599j

Labrador A, Cerenius Y, Svensson C, Theodor K, Plivelic T (2013) The yellow mini-hutch for SAXS experiments at MAX IV laboratory. J Phys: Conf Ser 425:072019. doi:10. 1088/1742-6596/425/7/072019

Lin N, Huang J, Dufresne A (2012) Preparation, properties and applications of polysaccharide nanocrystals in advanced functional nanomaterials: a review. Nanoscale 4:3274-3294. doi:10.1039/C2nr30260h

Lo Nostro P, Ninham BW (2012) Hofmeister phenomena: an update on ion specificity in biology. Chem Rev 112:2286-2322. doi:10.1021/cr200271j

Lyklema J (2003) Lyotropic sequences in colloid stability revisited. Adv Colloid Interface 100:1-12. doi:10.1016/ S0001-8686(02)00075-1

Lyklema J (2009) Simple Hofmeister series. Chem Phys Lett 467:217-222. doi:10.1016/J.Cplett.2008.11.013

Lyklema J (2013) Coagulation by multivalent counterions and the Schulze-Hardy rule. J Colloid Interface Sci 392:102-104. doi:10.1016/j.jcis.2012.09.066
Metropolis N, Rosenbluth AW, Rosenbluth MN, Teller AH, Teller E (1953) Equation of state calculations by fast computing machines. J Chem Phys 21:1087-1092. doi:10. $1063 / 1.1699114$

Michot LJ et al (2013) Coagulation of Na-montmorillonite by inorganic cations at neutral $\mathrm{pH}$. A combined transmission $\mathrm{X}$-ray microscopy, small angle and wide angle X-ray scattering study. Langmuir 29:3500-3510. doi:10.1021/ la400245n

Moon RJ, Martini A, Nairn J, Simonsen J, Youngblood J (2011) Cellulose nanomaterials review: structure, properties and nanocomposites. Chem Soc Rev 40:3941-3994. doi:10. 1039/C0CS00108B

Ninham BW, Lo Nostro P (2010) Molecular forces and self assembly in colloid, nano sciences and biology. Cambridge University Press, Cambridge

Peng BL, Dhar N, Liu HL, Tam KC (2011) Chemistry and applications of nanocrystalline cellulose and its derivatives: a nanotechnology perspective. Can J Chem Eng 89:1191-1206. doi:10.1002/cjce.20554

Rånby GB (1951) III. Fibrous macromolecular systems. Cellulose and muscle. The colloidal properties of cellulose micelles. Discuss Faraday Soc 11:158-164. doi:10.1039/ DF9511100158

Revol JF, Bradford H, Giasson J, Marchessault RH, Gray DG (1992) Helicoidal self-ordering of cellulose microfibrils in aqueous suspension. Int J Biol Macromol 14:170-172. doi:10.1016/S0141-8130(05)80008-X

Salas C, Nypelö T, Rodriguez-Abreu C, Carrillo C, Rojas OJ (2014) Nanocellulose properties and applications in colloids and interfaces. Curr Opin Colloid Interface Sci 19:383-396. doi:10.1016/j.cocis.2014.10.003

Sano M, Kamino A, Shinkai S (2001) Construction of carbon nanotube "stars" with dendrimers. Angew Chem Int Edit 40:4661. doi:10.1002/1521-3773(20011217)40:24<4661: Aid-Anie4661>3.0.Co;2-V

Sorensen CM (2001) Light scattering by fractal aggregates: a review. Aerosol Sci Technol 35:648-687. doi:10.1080/ 02786820117868

Stenqvist B, Thuresson A, Kurut A, Vacha R, Lund M (2013) Faunus-a flexible framework for Monte Carlo simulation. Mol Simul 39:1233-1239. doi:10.1080/08927022.2013. 828207

Vlachy N, Jagoda-Cwiklik B, Vácha R, Touraud D, Jungwirth P, Kunz W (2009) Hofmeister series and specific interactions of charged headgroups with aqueous ions. Adv Colloid Interface Sci 146:42-47. doi:10.1016/j.cis.2008.09.010

Wågberg L, Decher G, Norgren M, Lindström T, Ankerfors M, Axnäs K (2008) The build-up of polyelectrolyte multilayers of microfibrillated cellulose and cationic polyelectrolytes. Langmuir 24:784-795. doi:10.1021/la702481v

Zhong LX, Fu SY, Peng XW, Zhan HY, Sun RC (2012) Colloidal stability of negatively charged cellulose nanocrystalline in aqueous systems. Carbohydr Polym 90:644-649. doi:10.1016/j.carbpol.2012.05.091 